\title{
Symptomatic recovery in Miller Fisher syndrome parallels vestibular-perceptual and not vestibular-ocular reflex function
}

\author{
1 Academic Neuro-Otology, Centre for Neuroscience, Charing Cross Hospital, Imperial College London, London, UK \\ 2 Department of Ophthalmology, University Hospital Athens, Athens, Greece \\ ${ }^{3}$ Centre of Human and Aerospace Physiological Sciences, King's College London, London, UK \\ ${ }^{4}$ National Hospital for Neurology and Neurosurgery, Queen Square and Moorfields Eye Hospital, London, UK
}

Barry M. Seemungal ${ }^{1}$, Panos Masaoutis ${ }^{2}$, David A. Green ${ }^{3}$, Gordon T. Plant ${ }^{4}$ and Adolfo M. Bronstein

\section{Edited by:}

Dominik Straumann, University Hospital Zurich, Switzerland

\section{Reviewed by:}

Aasef G. Shaikh, Case Western

Reserve University, USA

Alexander A. Tarnutzer, University

Hospital Zurich, Switzerland

Mark F. Walker, Case Western Reserve

University, USA

*Correspondence:

Barry M. Seemungal, Charing Cross Hospital Campus, Room 10L16 (Lab Block), Fulham Palace Road, London W6 8RF, UK.

e-mail:b.seemungal@imperial.ac.uk

Unpleasant visual symptoms including oscillopsia and dizziness may occur when there is unexpected motion of the visual world across the subject's retina ("retinal slip") as in an acute spontaneous nystagmus or on head movement with an acute ophthalmoplegia. In contrast, subjects with chronic ocular dysmotility, e.g., congenital nystagmus or chronic progressive external ophthalmoplegia, are typically symptom free. The adaptive processes that render chronic patients asymptomatic are obscure but may include a suppression of oscillopsia perception as well as an increased tolerance to perceived oscillopsia. Such chronic asymptomatic patients display an attenuation of vestibular-mediated angular velocity perception, implying a possible contributory role in the adaptive process. In order to assess causality between symptoms, signs (i.e., eye movements), and vestibular-perceptual function, we prospectively assessed symptom ratings and ocular-motor and perceptual vestibular function, in a patient with acute but transient ophthalmoplegia due to Miller Fisher Syndrome (as a model of visuo-vestibular adaptation). The data show that perceptual measures of vestibular function display a significant attenuation as compared to ocular-motor measures during the acute, symptomatic period. Perhaps significantly, both symptomatic recovery and normalization of vestibular-perceptual function were delayed and then occurred in a parallel fashion. This is the first report showing that symptomatic recovery of visuo-vestibular symptoms is better paralleled by vestibular-perceptual testing than vestibular-ocular reflex (VOR) measures. The findings may have implications for the understanding of patients with chronic vestibular symptoms where VOR testing is often unhelpful.

Keywords: vestibular velocity storage mechanism, vestibular perception, acute ophthalmoplegia, Miller Fisher syndrome

\section{INTRODUCTION}

Congenital nystagmus subjects adapt to their continuous ocular oscillation such that the visual world is perceived as stable. Similarly, patients with chronic ophthalmoplegia are asymptomatic despite significant retinal motion of the visual world during head movement [due to loss of the vestibular-ocular reflex (VOR)-effected retinal image stabilization]. In contrast, patients with acute-onset nystagmus or ophthalmoplegia develop unpleasant and disorientating symptoms of oscillopsia (Leigh and Zee, 2006) and dizziness (Schabet, 2009) concomitant with their ocular dysmotility. That subjects with chronic dysmotility display an attenuation of vestibular function in terms of duration of sensation of dizziness with a whole-body rotation (Okada et al., 1999; Grunfeld et al., 2003) may suggest a possible mechanism underlying their beneficial adaptation. The duration of vestibular responses is in part modulated by the vestibular velocity storage mechanism, a brainstem-mediated mechanism that prolongs the duration of the vestibular signal as compared to that in primary vestibular afferent neurons. Velocity storage is thought to enhance the vestibular response to low frequency angular accelerations. That a suppression of the velocity storage mechanism may protect from symptoms of visuo-vestibular disorientation is supported by the finding that healthy humans, occupationally exposed to repetitive visual and vestibular motion stimulation, e.g., pilots and ballet dancers, also show an attenuated vestibular velocity storage (Aschan, 1954; Fukada et al., 1967). Thus one postulate may be that suppression of the velocity storage mechanism, may enable adaptation to conditions of abnormally intense or frequent, visuo-vestibular stimulation, either occupational or pathological.

The causality between attenuation of the vestibular velocity storage mechanism and (absence of) symptoms cannot be established in either chronic ophthalmoplegic patients or healthy volunteers, since both are asymptomatic. Additionally investigating patients with chronic ophthalmoplegia limits testing to perceptual measures of velocity storage since ocular motor measures (a surrogate measure of brainstem vestibular function) are untestable. Miller Fisher Syndrome (MFS), an uncommon post-infectious neurological condition, is an ideal model to answer many of these questions. First, MFS presents with an acute ophthalmoplegia leading to head movement induced visuo-vestibular disorientation, including oscillopsia and dizziness (Schabet, 2009). Second, the ophthalmoplegia is transient so the temporal process of recovery can be pursued with both ocular motor and perceptual measures of velocity storage and simultaneously assessing symptom burden. A temporal link with 
one or other measure of velocity storage with symptoms, and the sequence in which the various measures evolve, could allude to causality. Relevant prospective data during the 2-year clinical follow-up of a patient presenting with acute MFS was obtained.

\section{METHODS}

\section{CASE HISTORY}

A previously well 36-year-old man was referred for dizziness and oscillopsia. His oscillopsia and dizziness occurred on head movement and started 1 week following a brief flu-like illness. Examination 1 week after oscillopsia-onset showed complete ophthalmoplegia, limb areflexia, and ataxia. Investigations were unremarkable including brain MRI, nerve conduction studies, and anti-GQ $\mathrm{Q}_{1 \mathrm{~b}}$ antibodies. A lumbar puncture was declined. The patient remained severely ophthalmoparetic into the second week but the major part of the ophthalmoplegia resolved and he was no longer ataxic by the end of week 2 (i.e., full range of eye movements but gaze-evoked nystagmus when making eye movements beyond $30^{\circ}$ from the primary position and accompanied by diplopia) at which point he underwent laboratory assessment of vestibular-ocular and vestibular-perceptual function.

Subsequent testing took place over the 2-year period of clinical follow-up and consisted of laboratory and symptom assessment. We measured ocular-motor and perceptual measures of the velocity storage mechanism in response to whole-body angular velocity steps to the right and left using the method of Okada et al. (1999; Figure 1). Briefly, simultaneous VOR and perceptual responses were measured by their time constant (Tc) of exponential decline following a horizontal-plane rotational step stimulus (following $60 \mathrm{~s}$ of constant rotation at $90 \%$ to the right and left) in the dark. For each session, four stopping responses were obtained. Eye movement signals were measured by electro-oculography (EOG) and passed through an analogue low-pass filter (cut-off $30 \mathrm{~Hz}$ ) and digitally recorded at a $250 \mathrm{~Hz}$ sampling frequency. The EOG trace was calibrated in terms of voltage (or computer units) per angular excursion of the eye. The slow-phase velocity for each nystagmic beat was obtained by measuring the slope of the EOG trace. Perceptual responses were recorded by the subject turning a wheel congruent with his perceived self-rotation velocity. An output voltage propor- tional to the angular velocity of the wheel was digitally sampled and recorded at $250 \mathrm{~Hz}$. The perceptual output for each response was uncalibrated and thus was normalized for each response (where the peak output was converted to 100\%). The peak gain and exponential Tc of slow-phase velocity response of the VOR for each step acceleration was measured, and an average of four responses per session was obtained.

An exponential curve was fitted to the decay portion of both VOR and perceptual outputs (peak response to return to baseline) where the instantaneous value of the height of the fitted curve $y$, was related to time point $t$, by $y=P_{\mathrm{y}} \mathrm{e}^{-\mathrm{xt}}$, where $1 / x$ is the value of the Tc in seconds and $P_{\mathrm{y}}$ is the peak value of $y$ (in $\%$ for the ocular-motor response and in $100 \%$ for the perceptual response). The dominant Tc was derived from a maximized $r$ goodness of fit value (method of least squares) between the fitted Tc and original data.

To aid clinical assessment, the patient kept a personal diary, scoring his dizziness using the "Vertigo Symptom Scale" (Yardley et al., 1992; VSS: scores range 0-40 for the vertigo component of the questionnaire) and rating his visual symptoms from 0 to 10 which comprised two subscales: oscillopsia rated $0-5$ with $5=$ maximal oscillopsia as at the onset of his symptoms and diplopia, also rated $0-5$, with $5=$ continuous diplopia in all planes of gaze (the visual symptom score was then multiplied by two to allow the use of a single $y$-axis in Figure 3 for visual and vertigo scales).

\section{RESULTS}

Figure 2 shows representative raw data for ocular-motor and perceptual measures obtained from the laboratory sessions. Figure 3 shows the serial laboratory (upper panel: mean Tc and gain responses) and subjective (lower panel: symptoms) change over the follow-up period. During the first laboratory test (week 2 post-onset), the peak slow-phase ocular-motor responses were attenuated (abnormally low peak gain response) by a partial ophthalmoplegia (Figure 2; Ocular-motor "Session 1") but note the Tc of an exponential is independent of the peak response. Figure 3 shows an early suppression of the perceptual response in the face of maximal symptoms and remains suppressed for the duration of the symptomatic period. Testing over the subsequent 2 months (i.e., weeks 8-16) chart the variability of the ocular-motor Tc

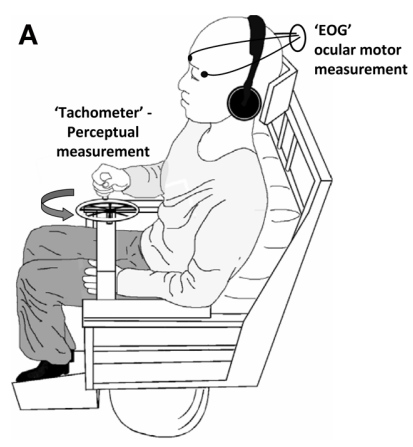

FIGURE 1 | Laboratory measurement of Vestibular function - The velocity storage mechanism. (A) Apparatus. Following a whole-body angular velocity step in the dark (and with white-noise sound masking), the ocular motor (i.e., Nystagmic) response is recorded by Electro-Oculography (EOG) and the perceptual response is obtained by subjects turning a wheel device ("Tachometer")

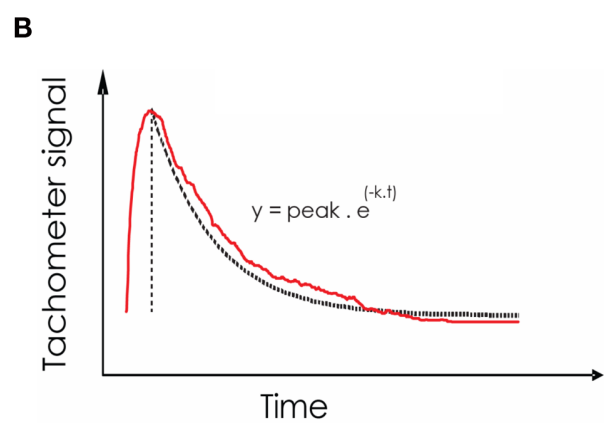

congruent with their sensation of turning. The device output voltage is proportional to the rotational velocity with which the wheel is turned. (B) Recorded signals. The decline in the perceptual response (following an initial upstroke from zero) is fitted to an exponential of time constant $(k)$. k equates to roughly $1 / 3$ the total duration of the signal. The ocular-motor slow-phase velocity also declines exponentially. 

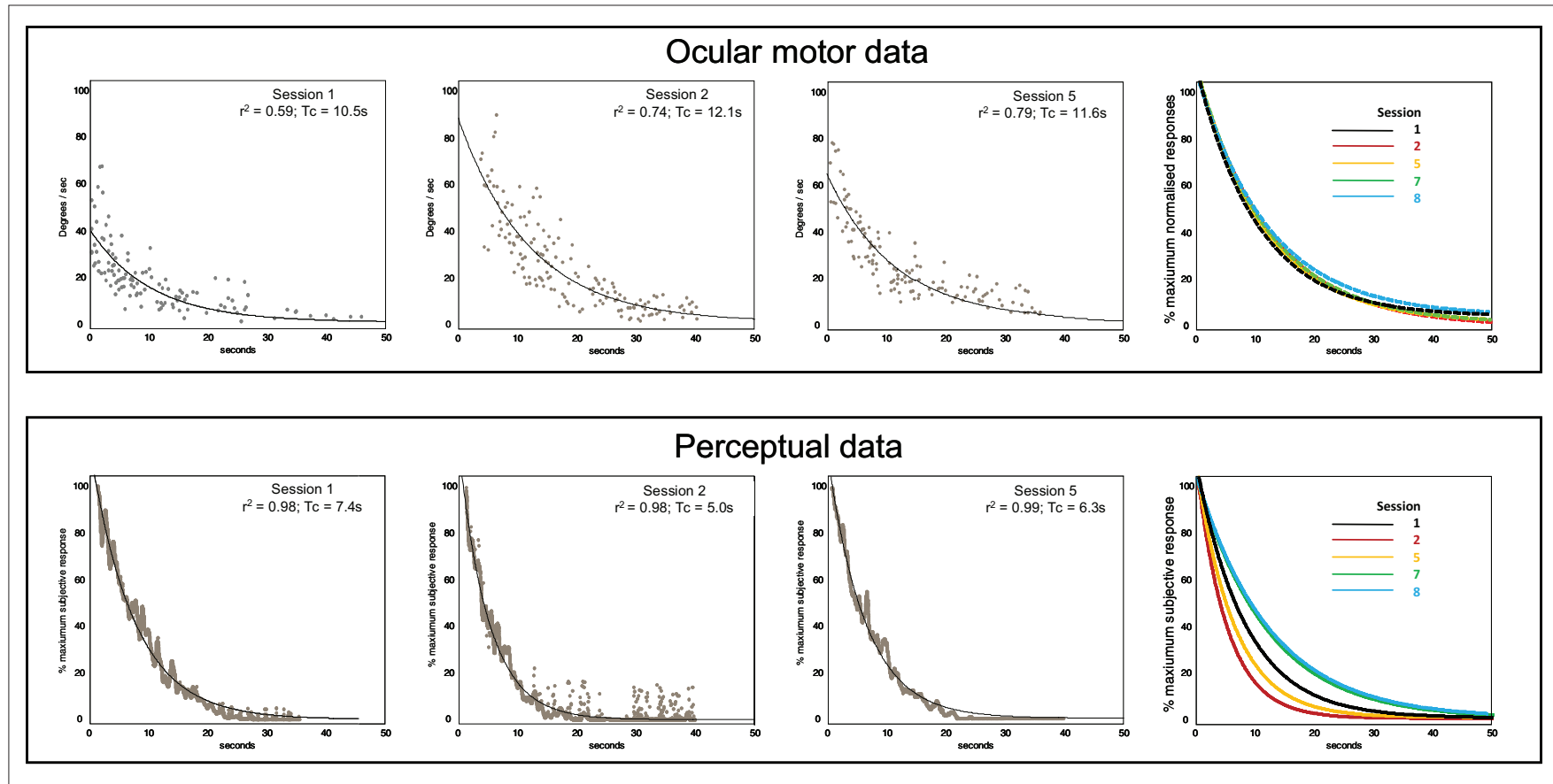

FIGURE 2 | Representative raw data. Upper panels - Ocular motor data. Data showing slow-phase velocities ( $\%$ s) following whole-body velocity steps, for sessions 1, 2, and 5. The far right panel shows fitted exponential plots for sessions 1, 2, 5, 7, and 8 (the peak slow-phase velocity was normalized here to $100 \%$ ). Lower panels - Perceptual data. Averaged tachometer signals (\% of the maximum sensed rotational velocity) and exponential fits for sessions 1 , 2 , and 5 . The far right panel shows exponential fits for sessions $1,2,5,7$, and 8 .

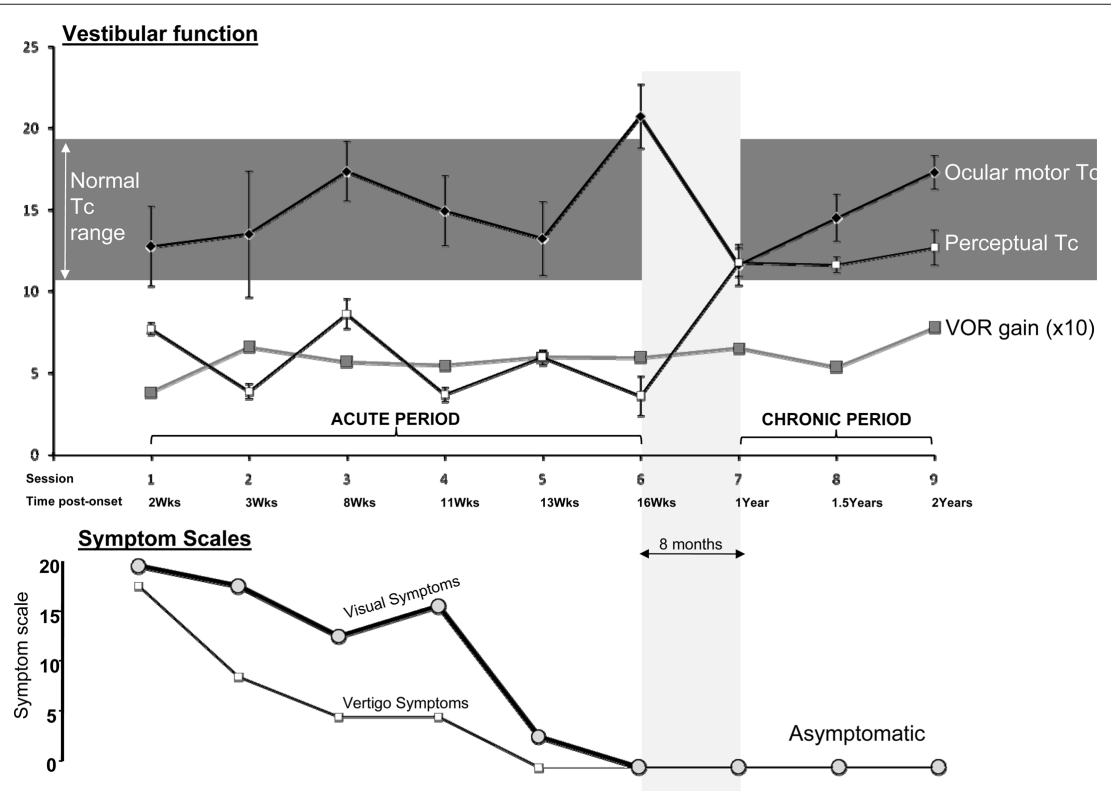

FIGURE 3 | Main results. Upper panel: Mean time constants (seconds) \pm 1 standard error, and mean peak VOR gains multiplied by 10 (10× peak slow-phase eye velocity/peak stimulus velocity). Gain was displayed as $\times 10$ to allow use of a single $y$-axis in the figure. Lower panel: The subjective symptom scales: Visual (thick line) and Vertigo Scales (thin line). Zero equals no symptoms. The central horizontal line in the figure ("Session/Time postonset") represents a common $x$-axis of time as labeled, for both upper and lower panels. response however this variability is confined to a normal range (Figure 3) in stark contrast to that for the perceptual response which remains consistently "suppressed." Note that perceptual responses are still abnormal at 16 weeks. Subsequent follow-up at 1 year found the patient to be entirely recovered. Note the reproducibility and similarity in magnitude of the perceptual 
and VOR measures over the final 12 months of follow-up. We also compared the mean Tc for the symptomatic ("Acute" sessions 1-6) and asymptomatic epochs ("Chronic" sessions 7-9) for both ocular-motor and perceptual Tcs. Four unpaired $t$-test comparisons were made (Bonferroni corrected significance of $P<0.0125)$. Acute versus chronic ocular-motor Tcs were not significantly different. In contrast perceptual Tcs were significantly lower $(P<0.0001)$ for the acute symptomatic versus the Chronic asymptomatic epochs. Comparing ocular-motor Tcs with perceptual Tcs, the eye movement measured Tc was longer than perceptual for the Acute epoch $(P<0.0001)$ but not significantly different for the Chronic epoch. In summary, the main finding was of a significantly different and shorter perceptual Tc during the Acute symptomatic phase.

\section{DISCUSSION}

We found that during recovery from an acute transient ophthalmoplegia, symptomatic recovery was better reflected in abnormal vestibular-perceptual function testing as compared to ocularmotor measures.

Prior studies of velocity storage in patients with nystagmus and ophthalmoplegia (Okada et al., 1999; Grunfeld et al., 2003) have only assessed post-adapted and hence asymptomatic subjects, in whom the process of adaptation is completed and hence rendered opaque. Indeed, in adapted subjects and healthy volunteers, perceptual and ocular-motor measures of the velocity storage mechanism are well correlated (Okada et al., 1999). This is congruent with the results in our patient for the late asymptomatic phase (see testing over year 2) but entirely at variance with the results in the initial symptomatic phase where we found a divergence between perceptual and ocular-motor measures of the velocity storage mechanism.

The observed link between visual and vestibular processing (Okada et al., 1999) may be explained by the convergence of both visual motion and vestibular signals onto secondary vestibular neurons in the brainstem vestibular nuclei (Waespe and Henn, 1977). Indeed this convergence is one route through which visual motion stimulation may engender sensations of self-motion as is commonly experienced when we see a nearby train move past. Since simplistically, the velocity storage mechanism can be considered to occur downstream of the secondary vestibular neurons, it follows that attenuating the velocity storage mechanism will also attenuate the effects of both inertially and visually driven vestibular signals in the brain. Consistent with this inference is the observation that in the laboratory, repetitive optokinetic or inertial stimulation induces a reduction in the duration of the velocity storage mechanism (Grunfeld et al., 2000b).

The current data however, cannot be entirely explained using the above model of a convergence of velocity storage processing confined to a brainstem locus. The observed perceptual-ocularmotor discrepancy in the acute symptomatic phase, suggests that the velocity storage mechanism for perception and the VOR, are mediated by neuronal circuits that are in part, non-overlapping. Although unknown, the cerebral cortex is the likely neuronal substrate mediating adaptation of the perceptual Tc. Interestingly cortical lesions can retard the return to normal values of the VOR velocity storage Tc following adaptation (Tusa et al., 1989). This suggests the intriguing possibility of a cortical modulation of both VOR and perceptual Tcs.

An additional finding of this report not available from chronic patient groups (Okada et al., 1999; Grunfeld et al., 2003) is the time course of adaptation to an acute disruption of visuo-vestibular synergy. That we found a shortened perceptual Tc on first testing implies that the perceptual response to an acute onset of visuovestibular mismatch occurs at least within 1-2 weeks of the insult. Although we do not have pre-morbid testing in this patient, data in healthy subjects (Clément et al., 2008) undergoing vestibular habituation (albeit in the dark) suggest that the perceptual Tc is in fact quicker to adapt than the VOR in responding to a visuovestibular mismatch. The relative lack of suppression of the VOR Tc in our patient on first testing may thus represent the fact that the ophthalmoplegia was transient and that a longer lasting ophthalmoplegia would have been required for an effect on VOR velocity storage Tcs to have been observed. The time course of a return to normal, at least in this patient, was of the order of 4 months. The "recovery" in the Tc for both VOR and perception in habituated healthy subjects is also of the order of several months (for both VOR and perceptual responses; Clément et al., 2008). At present however, it is unclear if suppression of the perceptual Tc is a response to symptoms or itself actively suppresses symptoms. That we observed a suppression of the perceptual Tc up until the point of symptom resolution suggests that suppressing the perceptual Tc may aid symptom resolution. Irrespective of the role of the perceptual Tc, previous data (Grunfeld et al., 2000a) suggest that an important component of patients' adaptation to retinal slip is the development of tolerance to residual perceptions of retinal slip, i.e., some patients continue to perceive some degree of retinal slip without experiencing unpleasant reactions.

The findings in our case may have implications for the study of mechanisms underlying symptoms in the chronic dizzy patient. In virtually all patients with a low-level vestibular insult such as a vestibular neuritis, lower-order adaptation occurs automatically as demonstrated by the cessation of nystagmus after a few days, even despite sustained unilateral peripheral vestibular loss. Some vestibular neuritis patients develop chronic symptoms dominated by sensitivity to visual motion stimulation (Bronstein, 2005), even in the face of recovered ocular-motor function (Palla et al., 2008). This contrasts with asymptomatic chronic ophthalmoplegia patients who have reduced sensitivity to visual motion (Acheson et al., 2001) which, in turn, is correlated to the degree of suppression of the vestibular-perceptual system (Grunfeld et al., 2003). The success of specific rehabilitation programs with optic flow methods (Pavlou et al., 2004) may involve retraining the brain whereby neural signals engendered by large field opto-kinetic stimuli are re-routed from vestibular-perceptual areas, whose activation engenders sensations of self-motion, to visual motion areas, whose activation is interpreted as motion of an external object (Dieterich and Brandt, 2008).

In summary, symptomatic recovery following a transient disturbance of the visuo-vestibular system (here due to a transient but complete, total external ophthalmoplegia), better parallels changes in high-order vestibular-perceptual mechanisms than lowerorder vestibular-ocular-motor mechanisms. This observation in 
retrospect is perhaps unsurprising given the abundance of clinical examples in which there is an uncoupling of ocular-motor and perceptual function; for example, patients with chronic dizziness display a poor correlation between VOR function and symptoms (Palla et al., 2008) and congenital nystagmus subjects may be entirely asymptomatic despite a dramatic eye oscillation (Leigh et al., 1988). Interestingly, congenital nystagmus subjects complain of oscillopsia when the retinal image is stabilized upon the retina (Leigh et al., 1988). This data provides evidence that central mechanisms are engaged in the active suppression of unpleasant sensations of oscillopsia in the face of even large amounts of retinal image motion.

\section{REFERENCES}

Acheson, J. F., Cassidy, L., Grunfeld, E. A., Shallo-Hoffman, J. A., and Bronstein, A. M. (2001). Elevated visual motion detection thresholds in adults with acquired ophthalmoplegia. $\mathrm{Br}$. J. Ophthalmol. 85, 1447-1449.

Aschan, G. (1954). Response to rotatory stimuli in fighter pilots. Acta Otolaryngol. Suppl. 116, 24-31.

Bronstein, A. (2005). Visual symptoms and vertigo. Neurol. Clin. 23, 705-713.

Clément, G., Tilikete, C., and Courjon, J. H. (2008). Retention of habituation of vestibulo-ocular reflex and sensation of rotation in humans. Exp. Brain Res. 190, 307-315.

Dieterich, M., and Brandt, T. (2008). Functional brain imaging of peripheral and central vestibular disorders. Brain 131, 2538-2552.

Fukada, T., Tokita, T., Aoki, S., Watanabe, T., Hishada, K., Tashhiro, K., Miyata, H., and Mazuoka, Y. (1967). Study on nystagmus during voluntary acts. Observations of rotation in ballet. ORL (Jpn.) S2, 11-12.
Grunfeld, E.A., Morland,A. B., Bronstein, A. M., and Gresty, M. A. (2000a). Adaptation to oscillopsia: a psychophysical and questionnaire investigation. Brain 123(Pt 2), 277-290.

Grunfeld,E.A.,Okada,T., Jáuregui-Renaud, K., and Bronstein, A. M. (2000b). The effect of habituation and plane of rotation on vestibular perceptual responses. J. Vestib. Res. 10, 193-200.

Grunfeld, E. A., Shallo-Hoffmann, J. A., Cassidy, L., Okada, T., Faldon, M., Acheson, J. F., and Bronstein, A. M. (2003). Vestibular perception in patients with acquired ophthalmoplegia. Neurology 60, 1993-1995.

Leigh, R. J., Dell'Osso, L. F., Yaniglos, S. S., and Thurston, S.E. (1988). Oscillopsia, retinal image stabilization and congenital nystagmus. Invest. Ophthalmol. Vis. Sci. 29, 279-282.

Leigh, R. J., and Zee, D. S. (2006). The Neurology of Eye Movements, 4th Edn. New York: OUP.

Okada, T., Grunfeld, E., Shallo-Hoffmann, J., and Bronstein, A. M. (1999). Vestibular perception of angular velocity in normal subjects and in

We suggest that vestibular-perceptual function testing may provide useful information when assessing symptoms and their response to treatment programs, in chronic dizziness.

\section{CONTRIBUTION}

All authors contributed to clinical and/or laboratory data acquisition. Barry M. Seemungal and Adolfo M. Bronstein were additionally responsible for all other aspects required for the realization of this report. Barry M. Seemungal is an Academy of Medical Sciences/ Health foundation Clinician Scientist. Adolfo M. Bronstein is supported by the Medical Research Council (UK).

patients with congenital nystagmus Brain 122(Pt 7), 1293-1303.

Palla, A., Straumann, D., and Bronstein, A M. (2008). Vestibular neuritis: vertigo and the high-acceleration vestibulo-ocular reflex. J. Neurol. 255, 1479-1482.

Pavlou, M., Lingeswaran, A., Davies, R. A., Gresty, M. A., and Bronstein, A. M. (2004). Simulator based rehabilitation in refractory dizziness. J. Neurol. 251, 983-995.

Schabet, M. (2009). Miller Fisher syndrome. Pract. Neurol. 9, 289-291.

Tusa, R. J., Demer, J. L., and Herdman, S. J. (1989). Cortical areas involved in OKN and VOR in cats: cortical lesions. J. Neurosci. 9, 1163-1178.

Waespe,W., and Henn, V.(1977). Neuronal activity in the vestibular nuclei of the alert monkey during vestibular and optokinetic stimulation. Exp. Brain Res. 27, 523-538.

Yardley, L., Masson, E., Verschuur, C. Haacke, N., and Luxon, L. (1992). Symptoms, anxiety and handicap in dizzy patients: development of the vertigo symptom scale. J. Psychosom Res. 36, 731-741.
Conflict of Interest Statement: The authors declare that the research was conducted in the absence of any commercial or financial relationships that could be construed as a potential conflict of interest.

Received: 14 September 2010; accepted: 12 January 2011; published online: 11 February 2011.

Citation: Seemungal BM, Masaoutis P, Green DA, Plant GT and Bronstein AM (2011) Symptomatic recovery in Miller Fisher syndrome parallels vestibularperceptual and not vestibular-ocular reflex function. Front. Neur. 2:2. doi: 10.3389 fneur.2011.00002

This article was submitted to Frontiers in Neuro-otology, a specialty of Frontiers in Neurology.

Copyright (c) 2011 Seemungal, Masaoutis, Green, Plant and Bronstein. This is an open-access article subject to an exclusive license agreement between the authors and Frontiers Media SA, which permits unrestricted use, distribution, and reproduction in any medium, provided the original authors and source are credited. 